\title{
MRS reports election results for 2015
}

www.mrs.org/governance

$\mathrm{M}$ embers of the Materials Research Society have elected a vice president (who will serve as president in 2016) and five directors to join the 2015 MRS Board of Directors. The Board is composed of the officers and 12 to 21 directors, established by Board resolution.

The officers of the Society are the president (who serves as chair of the Board), the vice president (who is also the president-elect), the secretary, the treas-urer (a position appointed by the Board of Directors), and the immediate past president. Terms of office expire at the end of the year indicated in parentheses. The asterisk (*) designates those who are newly elected. The annual election ended September 19, 2014.

The Board of Directors is organized into the following governing committees: Audit, External Relations, Finance, Governance, Nominating, Operational Oversight, and Planning. Chairs and Members of the committees are either designated by policy or are appointed by the president.

\author{
2015 MRS Officers \\ President \\ Oliver Kraft \\ Karlsruhe Institute of Technology \\ Immediate Past President \\ Tia Benson Tolle \\ The Boeing Company \\ Vice President (President-Elect) \\ *Kristi Anseth \\ University of Colorado Boulder \\ Secretary \\ Sean J. Hearne \\ Sandia National Laboratories \\ Treasurer \\ Michael R. Fitzsimmons \\ Los Alamos National Laboratory \\ Executive Director \\ Todd M. Osman \\ Materials Research Society

\section{Board of Directors \\ *Charles T. Black (2017) \\ Brookhaven National Laboratory} \\ Alexandra Boltasseva (2016) \\ Purdue University \\ C. Jeffrey Brinker (2016) \\ Sandia National Laboratories \\ and University of New Mexico
}

David Cahen (2015)

Weizmann Institute of Science

Stephen J. Eglash (2015)

Stanford University

Sossina M. Haile (2015)

California Institute of Technology

Andrea M. Hodge (2015)

University of Southern California

Hideo Hosono (2016)

Tokyo Institute of Technology

* Karen L. Kavanagh (2017)

Simon Fraser University

Fiona C. Meldrum (2015)

University of Leeds

Kornelius Nielsch (2016)

University of Hamburg

*Christine Ortiz (2017)

Massachusetts Institute of Technology

*Sabrina Sartori (2017)

University of Oslo

Eric A. Stach (2015)

Brookhaven National Laboratory

Loucas Tsakalakos (2016)

General Electric-Global Research Center

*Anke Weidenkaff (2017)

University of Stuttgart

19th International Conference on Microscopy of Semiconducting Materials http://msm2015.iopconfs.org

$\mathrm{T}$ he Institute of Physics (IoP) biannual conference series "Microscopy of Semiconducting Materials" (MSMXIX) will be held March 29-April 2, 2015, at Murray Edwards College, University of Cambridge, UK. The conference has a long tradition of focusing on the most recent advances in the study of the structural and electronic properties of semiconducting materials by the application of transmission and scanning electron microscopy. The latest developments in the use of other important micro-characterization techniques, including scanning probe microscopy and x-ray topography and diffraction, will also be featured. Developments in materials science and technology covering the complete range of elemental and compound semiconductors and nanostructures thereof will be described.

The conference is organized by the Electron Microscopy and Analysis Group, which includes members from physics, materials science, chemistry, and engineering disciplines.

Some of the symposium topics include analytical electron microscopy, cathodoluminescence, lattice defects in bulk materials, thin films, and scanning electron and ion-beam techniques.

Contributions for oral or poster presentations should be submitted online by December 29, 2014. There is an early registration deadline of February 25, 2015. The proceedings of the conference will be published in special issues of scientific journals. More information can be accessed at the meeting website at http:// msm2015.iopconfs.org. 


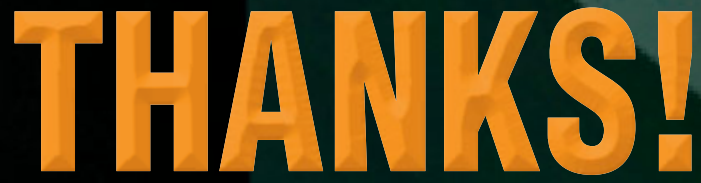

The following events at the 2014 MRS Fall Meeting have been sponsored, in part, by the generous contributions of these organizations

\section{POSTER SESSION REFRESHMENTS}

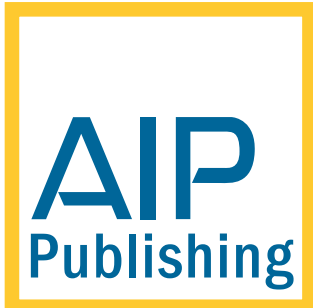

journals.aip.org/materials • Booth 117

\section{EVDOIK}

I N D USTR I E S

www.evonik.com

\section{COFFEE BREAKS}

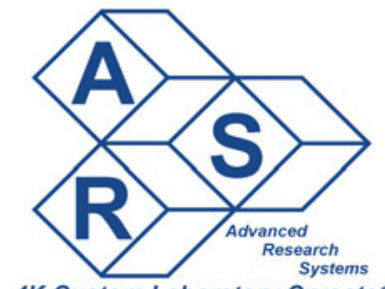

4K Custom Laboratory Cryostats

www.arscryo.com - Booth 310
WOMEN IN MATERIALS SCIENCE BREAKFAST

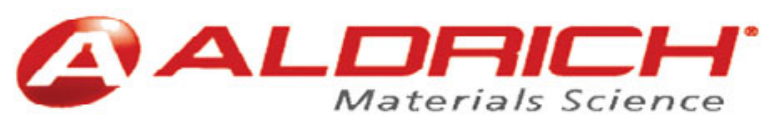

www.sigmaaldrich.com/matsci $\bullet$ Booth 600

\section{BADGE LANYARDS}

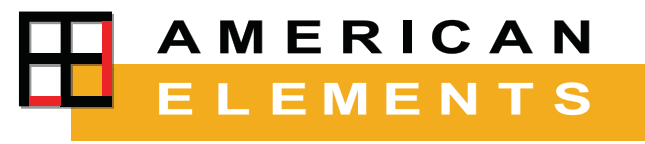

THE MATERIALS SCIENCE COMPANY ®

www.americanelements.com

\section{ACS Publications}

MOST TRUSTED. MOST CITED. MOST READ.

pubs.acs.org • Booth 123 


\section{XHIBITORS}

\section{HYNES CONVENTION CENTER • LEVEL 2}

Tuesday, December 2 11:00 am-5:30 pm

Wednesiay, December 3 11:00 am-5:30 pm

Thursday, December 4 10:00 am-1:30 pm

\section{T Analytik}

www.3t-analytik.com

qCellT Quartz Crystal Microbalance (QCM)

Sensor Instrument

A \& N Corporation

www.ancorp.com

Vacuum Components; Vacuum Chambers; Vacuum Valves

abcr GmbH

Booth 1312

www abcr de

Advanced Ceramic Powders; Metal-organics; Silanes

Across International LLC

Booth 308

www.acrossinternational.com

Lab Furnace; Induction Heaters; Vacuum Oven

ACS Publications

Booth 123

pubs acs.org

ACS Publications Materials Science Portfolio

AdNaNo Corp.

Booth 1102

www.adnano-tek.com

Pulsed Laser Deposition; UHV Sputter System;

Molecular Beam Epitaxy

AdValue Technology, LLC

Booth 620

www.advaluetech.com

Alumina and Quartz Crucibles, Tubes and Plates:

Quartz Cuvettes: Sapphire Crucibles

Advanced Energy Industries, Inc.

Booth 829

www.advanced-energy.com

Pinnacle ${ }^{\circledR}$ PLUS + HALO Power Supply; Paramount ${ }^{\circledR}$ RF

Power-Delivery Systems; Navigator ${ }^{\circledR}$ II Digital Matching

Network

Advanced Polymer Materials Inc. Booth 908

www.apmpolymers.com

Biodegradable Polymers; Functional Block Copolymers;

PEG Related Polymers

Advanced Research Systems, Inc.

Booth 310

www arscryo com

4K Cryocoolers; Custom Lab Systems;

Nanoscience Materials Characterization

\section{Advanced Test Reactor National}

Booth 222

Scientific User Facility

Idaho National Laboratory

atrnsuf.inl.gov

Cost-free Capabilities for Nuclear Materials Science
Aerotech, Inc.

www.aerotech.com

Booth 718

Q-Series Piezo Stages; Q-Lab Controller; ANT Stages

\section{AIP Publishing}

Booth 117

journals.aip.org

Physics Journals: Conference Proceedings;

Digital Archive

AIST-NT, Inc.

www.aist-nt.com

Atomic Force/Scanning Probe Microscopes; Combined

AFM \& Raman Spectroscopy Systems

\section{AIXTRON SE}

Booth 407

www.aixtron.com

MOCVD/CVD/PECVD Equipment; OVPD and PVPD

Equipment; ALD Equipment

AJA International, Inc.

Booth 800

www.ajaint.com

Sputtering Systems; Sputter Sources; Sputter Targets

\section{Aldrich Materials Science}

www.sigmaaldrich.com/matsci

Organic and Printed Electronics Materials; Nanomaterials

Materials for Energy and Electronics

Alfa Aesar, a Johnson Matthey Company Booth 912 www.alfa.com

High-Purity Metals; Evaporation Materials; Ceramics

$\begin{array}{ll}\text { Alfred University } & \text { Booth } 225 \\ \text { CACT.alfred.edu } & \end{array}$

Education; Research; Consulting

\section{Alicat Scientific, Inc.}

Booth 204

www.alicat.com

Mass Flow Meters: Mass Flow Controllers;

Pressure Controllers

\section{American Physical Society}

Booth 121

journals.aps.org

Publications; Physics Journals; Online Journals

ANA Innovation Huts and Services Inc. Booth 71 www.anarghyainnotech.com

Confocal Raman AFM; UHV ESCA/XPS/ARPES; UHV

Components and Systems; Cryo UHV AFM/STM; Mask

Aligners; Spincoaters
Anasys Instruments Corp.

www.anasysinstruments.com

Nanoscale Infrared Spectroscopy (nanolR, nanolR2)

AFM+; nano-TA2

Andeen-Hagerling, Inc.

Booth 421

www.andeen-hagerling.com

Capacitance/Loss Bridges; Capacitance Standards

Anfatec Instruments $A G$

Booth 1211

www.anfatec.de

SPM Controllers; LockIn Amplifiers; AFM/SNOM

\section{Angstrom Engineering Inc.}

Booth 928

wWw angstromengineering com

Thin Film, CVD, Sputter Deposition Systems; Electron Beam \& Thermal Evaporation Systems; R\&D and Pilot Production Systems: Robotic Cluster Tools

Angstrom Sciences Inc

Booth 1307

www.angstromsciences.com

Magnetrons; Sputtering Material; Sputtering Cathodes

Angstrom Scientific Inc.

Booth 721

www.angstrom.us

Electron Microscopy and Accessories; Nano-manipulators TEM Heating Holders

\section{Annealsys}

Booth 806

www.annealsys.com

RTP; DLI-CVD; MOCVD; ALD; RTCVD; LPCVD

\section{Anton Paar USA}

Booth 207

www.anton-paar.com

Small Angle X-Ray Scattering; SAXSpace; X-Ray Diffraction; Instrumented Indentation Testers; Scratch Testers;

Tribometer; Calotest/Calowear

Applied NanoStructures, Inc.

Booth 1206

www.appnano.com

SPM Probes; MEMS; Thermal Imaging

Applied Surface Technologies

Booth 1212

www.co2clean.com

$\mathrm{CO}_{2}$ Snow Jet Cleaning; Surface Cleaning

Substrate Cleaning

Applied Vacuum Technology, LLC

Booth 1207

www.appliedvacuum.com

UHV Chambers; UHV Flanges; Custom Components and

Assemblies 


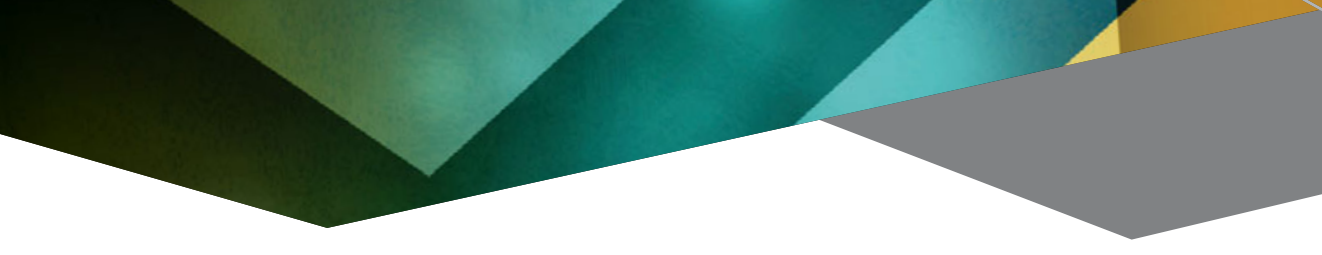

Aramco

Booth 317

www.aramcoservices.com

Advanced Materials Research; Membranes; Catalysis

Asahi Spectra Co., Ltd.

Booth 831

www.asahi-spectra.com

Xenon Light Source; Monochromatic Illuminator;

Optical Filters

\section{Asylum Research \\ an Oxford Instruments Company \\ www.AsylumResearch.com \\ Atomic Force/Scanning Probe Microscopes; \\ AFM/SPM Probes}

Booth 510

\section{attocube systems AG}

Booth 505

www.attocube.com

Piezo Positioning Systems; Low-vibration

Cryogen-free Cryostats; Low Temperature

Scanning Probe and Confocal Microscopes

\section{B\&W Tek, Inc.}

Booth 609

www bwtek com

Raman Spectrometers; Spectrometer Modules; Lasers

\section{Barnett Technical Services LLC}

Booth 1314

www.barnett-technical.com

Cathodoluminescence Systems; Micromanipulators;

Scanning Probe Microscopes

Bio-Logic USA, LLC

Booth 625

www bio-logic us

Potentiostat; Battery Cycler; Scanning Probe Microscopy

Blue Wave Semiconductors, Inc.

Booth 801

www.bluewavesemi.com

Substrate Wafer Heaters; Thin Film Deposition Systems;

Thin Films and Coating Materials; R\&D Services

\section{BNNT, LLC}

Booth 1124

www.bnnt.com

Boron Nitride Nanotubes

\section{Bose Corporation}

Booth 930

www bose-electroforce.com

DMA-Dynamic Fatigue Systems; Cardiovascular Stent Graft Test Systems; BioDynamic Tissue Engineering Test Systems

Brooks Automation, Inc.

Booth 200

www.brooks.com

Cryogenics; Cryopumps; Waterpumps; Cryochillers:

PCC Compact Coolers; Cryodyne Refrigerators

\section{Bruker}

Booth 30

www bruker.com

X-Ray Diffraction; Near Infrared Spectroscopy;

Raman Spectroscopy

\section{Bruker Nano Surfaces}

www bruker.com/nano

Atomic Force Microscopes; 3D Optical Microscopes; Stylus Profilers; Tribometers: Nano-, Micro- and Macro-Indenters;

Scratch Testers; AFM Probes

\section{Bruker AFM Probes}

Booth 304

www.BrukerAFMProbes.com

AFM Probes: SPM Probes; Imaging Accessories; Calibration and Reference Products
Materials Formulation

\section{Cambridge University Press}

www.cambridge.org/us/academic

Booth 100

Books; Journals

\section{Carl Zeiss Microscopy, LLC}

Booth 1016

www.zeiss.com/microscopy

Light Microscopes; Electron Microscopes;

$X$-ray Microscopes

Chemat Technology, Inc.

Booth 201

www.chemat.com

Spin Coating; Dip Coating; Chemical Precursors

ColdEdge Technologies, Inc.

Booth 608

www.coldedgetech.com

Cryostats; Cryocoolers; Cryogenics

CRAIC Technologies, Inc.

www.microspectra.com

Booth 1020

UV-visible-NIR microscopes; Microspectrophotometers;

Raman Microspectrometers

\section{CRC Press-Taylor \& Francis}

Booth 124

www crcpress.com

Books; Electronic Content

CRYO Industries of America, Inc.

Booth 423

www.cryoindustries.com

Liquid Helium Liquefiers; Closed Cycle Cryostats;

Continuous Flow Cryostats

\section{CrystalMaker Software Ltd.}

Booth 929

www.crystalmaker.com

CrystalMaker; CrystalDiffract; SingleCrystal

\section{CVD Equipment Corporation}

Booth 1213

www.crdequipment.com

CVD Equipment; Gas/Chemical Delivery Systems;

Gas Abatement Systems

\section{DCA Instruments, Inc.}

Booth 520

www.dca.fi

UHV PLD Systems; UHV Sputtering Systems;

MBE Systems

\section{De Gruyter}

Booth 1300

www.degruyter.com

Books; Journals; eBook; eJournals

\section{Delong America Inc.}

Booth 1022

www.lv-em.com

LVEM5 Electron Microscope

\section{Digital Surf SARL}

Booth 704

www.digitalsurf.com

Surface Imaging. Analysis and Metrology Software for

Microscopes and Profilers

\section{Dolcera}

Booth 623

www dolcera com

IP Analysis; Business Research; Product Development
Duniway Stockroom Corporation

Booth 601

www.duniway.com

Ion Pumps; Gauge Controllers; Pump Rebuilding Services

EBARA Technologies, Inc.

Booth 1023

www.ebaratech.com

Dry Vacuum Pumps; Turbomolecular Pumps

Exhaust Abatement

\section{Ecopia Corp.}

Booth 205

www.ecopia21.co.kr

Hall Effect Measurement Systems; RTP Systems

\section{EDAX Inc}

Booth 813

www.edax.com

Energy Dispersive X-ray; EBSD; X-ray Detectors

Electron Microscopy Sciences/Diatome Booth 316 www.emsdiasum.com

Grinders/Polishers; Diamond Wheels/Saws;

Microscopy Supplies

\section{Elsevier}

Booth 111

www.elsevier.com

Books; Journals; Electronic Products

Energetiq Technology, Inc.

Booth 709

www.energetiq.com

Laser-Driven Light Source

EPIR Technologies Inc.

www.epir.com

$\mathrm{HgCdTe}$ Wafers; Infrared Focal Plane Arrays:

Infrared R\&D Services

\section{FEI Company}

Booth 323

www fei.com

Talos TEM; Titan Themis; Teneo; Helios G3

Helios PFIB

\section{FemtoTools AG}

Booth 906

www.femtotools.com

Electro-Mechanical Probe Station; Micromechanical Testing System; Microrobotic Handling System

\section{Film Sense}

Booth 1120

www.film-sense.com

Ellipsometer

Fischione Instruments

Booth 506

www.fischione.com

TEM Accessories: Sample Preparation;

Specimen Holders

FlackTek, Inc.

Booth 804

www.speedmixer.com

Mixing Machines; Laboratory Mixers

High-speed Mixing

FUJIFILM Dimatix, Inc.

Booth 1003

www.dimatix.com

Dimatix Materials Printer; Dimatix Materials Cartridge;

Inkjet Printheads \& Systems

Furuya Metal Americas, Inc.

www.furuyametals.co.jp/english

Booth 920

Precious Metals Crucibles: Precious Metals Thermocouples;

Precious Metals Chemical Compounds 
Gamry Instruments

www.gamry.com

Potentiostats; Impedance Spectroscopy:

Quartz Crystal Microbalance

Gatan, Inc

Booth 412

www.gatan.com

Materials Characterization; Nanotechnology; Photovoltaics

\section{Geib Refining Corp.}

Booth 719

www geibrefining com

Precious Metal Reclaim; Gold and Platinum;

UHV Shield Cleaning

\section{Goodfellow Corporation}

Booth 1013

www.goodfellowusa.com

Metals and Materials for Research \& Development

Graphene Square Inc.

Booth 729

www.graphenesquare.com

Customizable CVD Equipment; Graphene Samples on a

Variety of Substrates; Other Graphene Derivatives

\section{Hamamatsu Corporation}

Booth 603

www.sales.hamamatsu.com

Quantum Yield Spectrometer; NIR Photomultipliers and

Detectors; VUV; Imaging

\section{Harvard University}

Booth 1208

\section{Center for Nanoscale Systems}

cns.fas.harvard.edu

Shared Facilities Center for Nanotechnology; NNIN Site

\begin{tabular}{ll}
\hline HeatWave Labs Inc. & Booth 703
\end{tabular}

www.cathode.com

Substrate Heaters; Electron Guns; Temperature Controllers

\section{Heidelberg Instruments, Inc.}

www.himt.de

Booth 523

microPG Systems; Direct Write Lithography Systems;

Maskless Lithography Tools

HEKA Instruments Inc.

Booth 819

www.heka.com

Electrochemical Scanning Microscopes; Potentiostats

\section{Herzan LLC}

Booth 322

www.herzan.com

AEK-2002 Acoustic Enclosure; TS Vibration Isolation

Tables; WaveCatcher Site Survey Tool

\section{Hiden Isochema Ltd.}

Booth 1129

www hidenisochema com

Manometric Gas Sorption Analyzers;

Gas and Vapor Sorption Analyzers

Hitachi High Technologies

Booth 1117

America, Inc.

www.hitachi-hta.com

Scanning Electron Microscopes: Transmission Electron

Microscopes; Focused Ion Beam Scanning Electron

Microscopes

\section{HORIBA Scientific}

Booth 917

www.horiba.com/scientific

Spectroscopy/Spectrometers; Raman; Ellipsometry
Hummingbird Scientific

www.hummingbirdscientific.com

In Situ TEM Holders; TEM Holders

Environmental Holders

\section{Huntington Mechanical}

Booth 1000

Laboratories, Inc.

www.huntvac.com

Feedthroughs; Valves; Chambers

Hysitron, Inc.

Booth 417

www.hysitron.com

TI 950 Tribolndenter; TI Premier;

PI Series Picolndenter

\section{Image Metrology A/S}

www.imagemet.com

SPIP Image Analysis Software

\section{Impedans Ltd.}

www.impedans.com

Langmuir System; Semion System; Octiv Systems

Inel, Inc.

Booth 324

www.inel.us

X-ray Diffractometers; Powder Diffractomers:

X-ray Diffraction

\section{INFICON}

Booth 525

www.inficon.com

Thin Film Deposition; Quartz Crystal Microbalance

Research; Vacuum Gauges and Components

Innovative Technology, Inc.

Booth 725

www.gloveboxes.com

Glove Box Systems: Gas Purifiers:

Solvent Purification Systems

\section{Integrated Dynamics Engineering}

Booth 93

www.ideworld.com

Vibration Isolation; EMI Cancellation

Acoustic Control

\section{International Centre for}

Diffraction Data (ICDD)

www.icdd.com

X-Ray Powder Diffraction; Database; Software

International Union of Crystallography Booth 114

www.iucr.org

Journals; Reference Books; Online Services

\section{Intlvac Thin Film}

Booth 728

www.intlvac.com

Ion Beam Etch Systems; Physical Vapor Deposition

\section{IOP Publishing}

Booth 104

ioppublishing.org

Journals, magazines and ebooks covering materials

research, applied physics, nanoscience and novel applications

\section{J microTechnology, Inc.}

Booth 905

\section{www.jmicrotechnology.com}

Probe Stations; Probe Positioners; Test Station
Janis Research Company, LLC

Booth 406

www.janis.com

Micromanipulated Probe Stations; Cryostats; Cryocoolers

\section{Japan Advanced Institute}

of Science and Technology

www.jaist.ac.jp/ms/english/index.htm

International Education; Advanced Research

Graduate School

Japan Science and Technology Agency Booth 217 www.jst.go.jp/tt/EN/

Novel Materials and Technologies for Licensing

Japan Society of Applied Physics

Booth 108

www.jsap.or.jp/english/index.htm

Journals

\section{JASCO}

Booth 924

www.jascoinc.com

Raman; Portable Raman; FTIR Microscopes

Near Field Microscopy; Ellipsometry

JEOL USA, Inc.

Booth 413

www.jeolusa.com

ARM 200/300 TEM; SEM; Auger/MicroProbe

JoVE

Booth 109

www.jove.com

Peer-reviewed Scientific Video Journal;

Science Education Database

\section{JPK Instruments AG}

Booth 521

www.jpk.com

Atomic Force Microscopy; Optical Tweezers;

Cell Mechanics

k-Space Associates, Inc.

www.k-space.com

Real-time Measurement of Substrate Temperature;

Thin-film Thickness and Deposition Rate; Film Stress

and Wafer Curvature

Kaufman \& Robinson, Inc.

Booth 807

www.ionsources.com

Ion Sources; Electron Sources; Power Supplies

Keithley Instruments, Inc.

www.keithley.com

Source Measure Unit Instruments; Low Level Electrica

Measurement Instruments; I-V Characterization Systems

DMMs and Power Supplies

\section{Keysight Technologies}

Booth 401

www.keysight.com/find/nano

Atomic Force, Scanning Probe, Field Emission

Scanning Electron and Scanning Electron Microscopes;

Nanomechanical Testing Systems

KLA-Tencor Corporation

Booth 616

www kla-tencor.com

Stylus Profiler; Universal Microscope

KP Technology USA Inc.

www.kelvinprobe.com

Air Photoemission, Scanning and UHV Kelvin

Probe Systems; Corrosion 
Kurt J. Lesker Company

Booth 400

www.lesker.com

Pure Targets and Materials; Vacuum Components; Thin

Film Deposition Systems; Target Bonding Services; UHV

Manipulation; Vacuum Valves

\section{Lab Synergy, LLC}

labsynergy.com

Particle Size Analysis; Nano Milling and Sizing;

Mechanical Alloying

\section{Labtec Sales Partners LLC}

Booth 604

www.labtecsp.com

Maskless Lithography Systems; Deposition Systems;

Etching Systems

\section{Lake Shore Cryotronics, Inc.}

Booth 701

www.lakeshore.com

Probe Stations; Hall Effect Systems; Cryogenic Instruments and Sensors

Lambda Technologies, Inc.

Booth 702

www.microcure.com

Broadband Microwave Laboratory Oven; Variable Frequency

Microwave Tools; Microwave Process Development Services

\section{Landt Instruments, Inc.}

Booth 1118

www landtinst.com

Battery Testing Systems

\section{Lehighton Electronics Inc.}

Booth 909

www lehighton com

Metrology Process Control Instruments; Sheet Resistance;

Electrical Mobility

\section{Leica Microsystems Inc.}

Booth 517

\section{www.leica-microsystems.com}

Microscopes; Sample Preparation; Imaging Systems

\section{Logitech Ltd.}

Booth 723

www.logitech.uk.com

Akribis-air Intelligent Sample Preparation System; CMP.

CP4000 Chemical Polishing System

\section{Braun, Inc.}

Booth 700

www.mbraunusa.com

Gloveboxes \& Gas Purifiers; Vacuum Systems; Solvent

Purification Systems

\section{MANTIS Deposition Ltd}

Booth 910

www mantisdeposition com

Nanoparticle Sources; UHV Deposition; PVD

McPherson, Inc.

Booth 1127

www.mcphersoninc.com

Spectroscopy Systems; Vacuum Spectrometers;

Soft X-ray; Raman

\section{Mettler-Toledo, LLC}

Booth 605

www. mt com

DSC; TGA; Flash DSC; DMA; TMA; Microscopy Hot Stage

\section{Micro Photonics Inc.}

www microphotonics com

Booth 305

Micro CT; X-ray Cameras; ø X-ray Diffraction
MicroFab Technologies, Inc.

Booth 904

www.microfab.com

Inkjet Printing Systems; Printed Electronics;

3-D Printing

\section{Microtrac Inc.}

Booth 828

www.microtrac.com

Particle Analyzers; Image Analyzers; Surface Area

Microwave Enterprises, Ltd.

Booth 1116

www.mwe-Itd.com

Microwave Plasma CVD Systems; Diamond Deposition

Systems; Single Crystal and Polycrystalline Lab Grown

Diamond Material

\begin{tabular}{ll}
\hline MicroXact Inc. & Booth $\mathbf{8 0 9}$ \\
www.microxact.com & \\
Probe Systems &
\end{tabular}

Probe Systems

\section{MIDAS Mask Aligners}

Booth 722

www.midasus.com

Mask Aligners; Spin Coaters; Scanning Electron

Microscopes

\section{MKS Instruments}

Booth 918

wWW.mksinst.com

Vacuum Quality Monitor; Vacuum Gauges and Components: Mass Flow Controllers

\section{MMR Technologies, Inc.}

Booth 1001

www.mmr-tech com

elan2 Liquid Nitrogen Generators; Variable Temperature

Hall \& Seebeck Measurement Systems

\section{MTI Corporation}

Booth 901

www.mtixtl.com

Battery R\&D Equipment; High Temperature Furnaces:

Single Crystal Substrates

Multiwire Laboratories, Ltd.

Booth 1007

www.multiwire.com

Real-Time Back-Reflection Laue X-ray Camera System

\section{NanoAndMore USA Inc.}

Booth 610

www.nanoandmore.com

AFM and SPM Probes: Particle Size and Zeta Potentia

Analyzers; DIHM

\section{NANOLANE}

Booth 808

www nano-lane com

Sarfus Mapping Stations; SEEC Slide

NanoMagnetics Instruments USA, LLC Booth 1101

www nanomagnetics-inst com

AFM/SPM; Scanning Hall Probe; Cryostats

Nanomechanics, Inc.

Booth 522

www. insem com

Nanoindenter

\section{Nanonics Imaging Ltd.}

Booth 817

www nanonics. co.i

NSOM/SNOM/SPM; AFM/Raman/TERS

Multiprobe Systems

\section{Nanoscribe GmbH}

Booth 1021

www.nanoscribe.de

3D Laser Lithography Systems; Photoresists
Nanosurf, Inc.

Booth 1010

www.nanosurf.com

Atomic Force Microscope; FluidFM; ARTIDIS

\section{Nanounity}

Booth 706

www.nanounity.com

SEM; Raman; Nanoindentation

National Electrostatics Corp.

Booth 409

www.pelletron.com

Pelletron Accelerator; RBS/PIXE/ERD; Accelerator Mass

Spectrometry

\section{National Nanotechnology}

Booth 1304

Infrastructure Network

www.nnin.org

Nanofabrication Services: Nanofabrication

User Facility

National Reconnaissance Office

Booth 224

dii.westfields.net

Aerospace; Remote Sensing: Research \& Development

Funding

\section{Nature Publishing Group}

Booth 110

www.nature.com

Medical \& Scientific Journals

NaugaNeedles LLC

Booth 519

www.nauganeedles.com

AFM Probes; STM Probes; Micro Nano Device

NBM Design, Inc

Booth 822

www.nbmdesign.com

Pulsed Laser Deposition Systems; Deposition Technologies Custom Vacuum Systems

Neaspec GmbH

Booth 509

www.neaspec.com

nano-FTIR; Near-field Microscopy; Nano-Spectroscopy

\section{Neocera, LLC}

Booth 716

www.neocera.com

Pulsed Laser Deposition Systems; Pulsed Electron

Deposition Systems

\section{Netzsch Instruments N.A. LLC}

Booth 1024

www netzsch-thermal-analysis com

Thermal Analysis; Thermal Conductivity;

Thermal Expansion; Calorimetry

\section{NIL Technology ApS}

Booth 921

www.nilt.com

Nanoimprint Tools; Masters for Nanostructure Replication;

Nanolithography and Processing

Nissan Chemical Industries, Lto.

Booth 1313

www.nissanchem.co.jp

Aptamer; 3D Micro Fluidics; Bio-compatible Coating

\section{NIS}

Booth 21

www.nist.gov/srm

Standard Reference Materials; Data and

Calibration Services 
NIST/CNST

Booth 220

www.cnst.nist.gov

Nanoscale Research Program; Nanofabrication Facility:

User Facility

Nor-Cal Products, Inc.

Booth 425

www.n-C.com

Vacuum Chambers; Vacuum Valves; Vacuum Components

\section{Northeastern University}

Booth 1303

Department of Chemical Engineering

www.che.neu.edu

MS Graduate Programs; PhD Graduate Programs;

Faculty Recruitment

\section{NovaCentrix}

Booth 1111

www.novacentrix.com

PulseForge Equipment; Metalon and PChem Conductive Inks; Printed Electronics; Flashlamp

\section{NT-MDT Co.}

Booth 210

www.ntmdt.us

SPM/AFM/STM; Raman TERS; Spectroscopy

\section{Nuts and Bolts Publications}

Booth 1200

www. nutsandboltsmaterials.com

Entrepreneur Textbooks

\section{Oerlikon Leybold Vacuum, USA}

Booth 1306

www.oerlikon.com/leyboldvacuum

PVD and ALD Equipment; Vacuum Pumps

Leak Detectors

\section{Olympus}

Booth 320

www olympus-ims.com

Inverted Metallographs; Upright Microscopes

Stereo Microscopes; Filter Inspection

\section{Olympus Corp.}

Booth 619

probe.olympus-global.com/en/

Micro Cantilevers for Scanning Probe Microscopy

Optofluidics, Inc.

Booth 1125

www.opfluid.com

Nanoparticle; Optical Tweezers; Photonics; Materials

Analysis; Nanotechnology

\section{OptiGrate Corporation}

Booth 1209

www.optigrate.com

Volume Bragg Gratings; Ultra-Narrow-Band Notch Filter

BragGrate BandPass Filters

\section{OriginLab Corporation}

Booth 1030

www.originlab.com

Origin Software; OriginPro Software

\section{Oxford Instruments}

Booth 511

www.oxford-instruments.com

AZtec Microanalysis: PlasmaPro; Triton; M700 Helium

Compressor; Scanning Probe Microscopy; Electron

Spectroscopy; Cypher AFM; Thin Film and Tailored UHV

Systems

\section{Oxford University Press}

Booth 112

www.oup.com/us

Publications
PANalytical Inc.

Booth 900 www.panalytical.com

X-ray Diffraction; Computed Tomography; Small Angle X-ray Scattering; X-ray Fluorescence

Park Systems Inc.

Booth 311

www.parkafm.com

Atomic Force Microscopes-Park NX10, Park NX20 and Park XE7

\section{Particle Sizing Systems}

Booth 710

www.pssnicomp.com

Particle Size; Zeta Potential; Particle Counting

\section{Pascal Technologies Inc.}

www.pascaltechnologies.com

Vacuum Systems; Vacuum Equipment; Leak Detectors

\section{Physics Today}

Booth 119

www.physicstoday.org

Publications; Buyers Guide

PicoQuant Photonics North America Inc. Booth 1205 www.picoquant-usa.com

Laser Sources; Photon Counting Electronics

Plasmaterials, Inc.

Booth 524

www. plasmaterials. com

Sputtering Targets; Evaporation Materials; Backing Plates; Bonding

\section{PROTO Manufacturing}

Booth 820

\section{www.protoxrd.com}

Powder Diffraction; Laue Single Crystal Orientation;

$X$-ray Tubes

Protochips, Inc.

www.protochips.com

Aduro Heating and Electrical In Situ Holder; Poseido Liquid and Electrochemistry Holders; C-flat Holey Carbon Specimen Support Grids

\section{PVD Products, Inc.}

www.pvdproducts.com

Pulsed Laser Deposition Systems; Sputtering Systems

Evaporation Systems

\section{Quantachrome Instruments}

www.quantachrome.com

Gas Sorption; Pycnometers; Porometers; Porosimeters Vapor Sorption; Water Sorption; Rotary Riffle; Autotap

\section{Quantum Design, Inc.}

Booth 501 www.qdusa.com

He Liquefier; Physical Property Measurement System; Squid Magnetometer; Crystal Furnace

\section{R.D. Mathis Company}

Booth 617

www.rdmathis.com

Evaporation Sources; Evaporation Materials;

Power Supplies

Radiant Technologies, Inc

Booth 903

www.ferroelectrictesters.com

Ferroelectric Testers; MultiFerroic Testers; Piezoelectric

Test Systems
Refining Systems, Inc.

www.refiningsystems.com

Sputtering Targets; Crucibles and Dishes; Evaporation

Materials; Wires and Tubing

\section{Renishaw Inc.}

Booth 411

www.renishaw.com

Raman Microscopes; Spectrometers

RHK Technology, Inc.

Booth 612

www.rhk-tech.com

SPM Universal Controls; UHV STM; UHV AFM/STM;

TERS/Nano-Optics

Rigaku Americas Corporation

Booth 712

www.rigaku.com

MiniFlex 600

Rocky Mountain Vacuum Tech, Inc.

Booth 306

www.rmvac.com

Vacuum Equipment; Vacuum Components

Royal Society of Chemistry

Booth 1301

www.rsc.org

Journals; Books; Databases; Membership

SAGE Publications

www.sagepub.com

Books; Journals

Scientific Instruments, Inc

www.scientificinstruments.com

9700 Controller; SCM10 Indicator; Diodes

Seki Diamond Systems

www.sekidiamond.com

Microwave Plasma CVD Systems; Hot Filament CVD

Systems; Plasma CVD Systems; Thermal CVD Systems

Diamond Materials; Thermal Conductivity Measurement

SemiconSoft, Inc.

Booth 907

www.semiconsoft.com

Thickness Measurement; Polymer Thickness;

Spectroscopic Reflectometer

Semicore Equipment Inc.

www semicore com

Sputtering Systems; Evaporation Systems; Custom PVD

Systems \& Components

ShenZhen Nanotech Port Co., Ltd.

Booth 1112

www.nanotubes.com.cn

Carbon Nanotubes; CNT Conductive Paste;

CNT Masterbatch

Sixth Element (Changzhou) Materials

Booth 811 Technology Co. Ltd.

www thesixthelement com cn

Graphene; Graphene Oxide

SmarAct Inc

Booth 821

www.smaract.com

Micropositioners; Nanopositioners; Parallel Kinematis

Solartron Analytical (AMETEK)

Booth 812

www.solartronanalytical.com

Materials Test Systems 
Sonaer, Inc.

Booth 622

www.sonaer.com

Ultrasonic Atomizer Nozzles; Robotic Coating Systems;

Ultrasonic Particle Generators

\section{SonoPlot, Inc.}

www.sonoplot.com

Microplotter Materials Dispensing Systems; Printed

Electronics; Materials Printer

SPECS Surface Nano Analysis, Inc.

Booth 203

www.specs.com

JT Scanning Tunneling Microscope; NAP-XPS; LEEM-PEEM

SPI Supplies/Structure Probe, Inc.

Booth 1203

www.2spi.com

Plasma Etching Systems; Graphene Products;

Ion Milling Systems

\section{Springer}

Booth 101

www.springer.com

Books; Journals; E-Books

STAIB Instruments, Inc.

Booth 717

www staibinstruments com

RHEED; Auger; Surface Analysis

Strem Chemicals, Inc.

Booth 1012

www strem com

Nanomaterials; CVD/ALD Precursors; Bubblers

Super Conductor Materials, Inc.

Booth 602

www.scm-inc.com

Sputtering Targets; Evaporation Materials;

Crucible Liners

\section{Surface Measurement Systems}

Booth 621

www.surfacemeasurementsystems.com

Gravimetric Vapor Sorption; Surface Energy Analyzer;

Dynamic Vapor Sorption

\section{SVT Associates, Inc.}

Booth 410

www.svta.com

Molecular Beam Epitaxy; Pulsed Laser Deposition;

Atomic Layer Deposition

SwissLitho AG

Booth 1122

www.swisslitho.com

NanoFrazor Explore

Synton-MDP AG

Booth 1108

www.synton-mdp.ch

Diamond Tips; Nanoindenter Tips; Stylus Tips

T\&C Power Conversion, Inc

Booth 1210 www.tcpowerconversion.com

$13.56 \mathrm{MHz}$ Complete RF Delivery Systems from 100W up to 2400W; LF Generators; Custom RF Amplifiers

\section{TA Instruments}

Booth 823

www tainstruments com

Discovery DSC; Discovery Hybrid Rheometer; DLF-1200

Tamura Corporation

Booth 922
TDC Corporation

www.mirror-polish.com

Polishing/Lapping Service; Precision Manufacturing

Super Precision Polished Products

\section{Ted Pella, Inc.}

www.tedpella.com

Microscopy Supplies; Sample Prep; Vacuum Coating

\section{Tekna Plasma Systems Inc.}

www.tekna.com

Integrated Plasma Systems

\section{Telemark}

Booth 824

www.telemark.com

Electron Beam Deposition; Film Thickness Monitoring;

Ion Beam Sources

\section{Tescan USA Inc}

Booth 816

www.tescan-usa.com

Scanning Electron Microscopes; Focused Ion Beam

Workstations; Mineral Analyzers

The Mellen Company, Inc.

Booth 805

www mellencompany com

Furnaces; Control Systems; Heaters

\section{Thermo Scientific}

Booth 422

www thermoscientific com/carbon

Raman Imaging Microscope; XPS Spectrometer

$X$-Ray Microanalysis System

Toho Technology Inc.

www.tohotechnology.com

FLX; TohoSpec 3100; NanoSpec II; RPM-Blue; HL5500;

Vertex

\section{Trion Technology, Inc.}

www.triontech.com

Plasma Etch Equipment: Plasma Deposition Equipment; Photoresist Strip Equipment

\section{UES, Inc.}

Booth 606

www.ues.com

RoboMet.3D Serial Sectioning System

Advanced Coatings for Die Casting

\section{Ultratech/Cambridge NanoTech}

Booth 708

www.cambridgenanotechALD.com

ALD Systems Deposition Systems; ALD Deposition Tools;

Coating Services

ULVAC Technologies, Inc.

Booth 713

www.ulvac.com

Nano Particle Deposition Systems: Rapid Thermal Annealing

Equipment; Thermoelectric Measuring Equipment

United Mineral \& Chemical Corporation Booth 1311 www.umccorp.com

MBE Source Materials; MBE Equipment; Dopants

Vacuum Technology Inc.

Booth 1029

www.vti-glovebox.com

Glove Box; Gas Purification System; Evaporator
VAT, Inc.

Booth 325

Www.vatvalve.com

Vacuum Valves; Control Valves; Leak Valves

Verder Scientific, Inc.

Booth 923

www.verder-scientific.com

Laboratory Milling \& Sieving

VG Scienta, Inc.

Booth 1008

www.vgscienta.com

Photoelectron Spectroscopy; Molecular Beam Epitaxy;

Scanning Probe Microscop

VG Scienta Ltd.

Booth 1310

www vgscienta com

Ultra High Vacuum; Manipulation; Linear and Rotary Drives

Transfer

Vigor Gas Purification Technologies Inc. Booth 825 www.vigor-glovebox.com

Glove Box: Gas Purification System; Solvent Purification System

Wafer World Inc.

Booth 1005

www waferworld com

Silicon Wafers; Germanium Wafers; Clean Room Packaging

Wiley

Booth 116

www.wiley.com

Books; Journals; Online Resources

WITec Instruments Corp.

www.witec-instruments.com

Confocal Raman Microscopy; Scanning Near-Field

Microscopy; Atomic Force Microscopy

J.A. Woollam Company, Inc.

Booth 1002

www.jawoollam.com

Spectroscopic Ellipsometers

World Scientific Publishing Co.

Booth 120

www.worldscientific.com

Books/eBooks; Journals/eJournals

XEI Scientific, Inc.

www.evactron.com

Remote Plasma De-contaminators for SEM, FIB and High Vacuum Chambers; Sample Precleaning Systems; New EP Low-cost Plasma Cleane

Yugyokuen Ceramics Co., Ltd.

Booth 307

www yugyokuen-ceramics.com

Ceramic; Vacuum Components; Brazing

Zurich Instruments Ltd.

Booth 1128

www.zhinst.com

HF2LI Lock-in Amplifier; UHFLI Lock-in Amplifier;

UHF-BOX Boxcar Averager

Zygo Corporation

Booth 913

www.zygo.com

Metrology; Optics; Testing Service

www tamura-ss co.jp

Gallium Oxide Substrates 


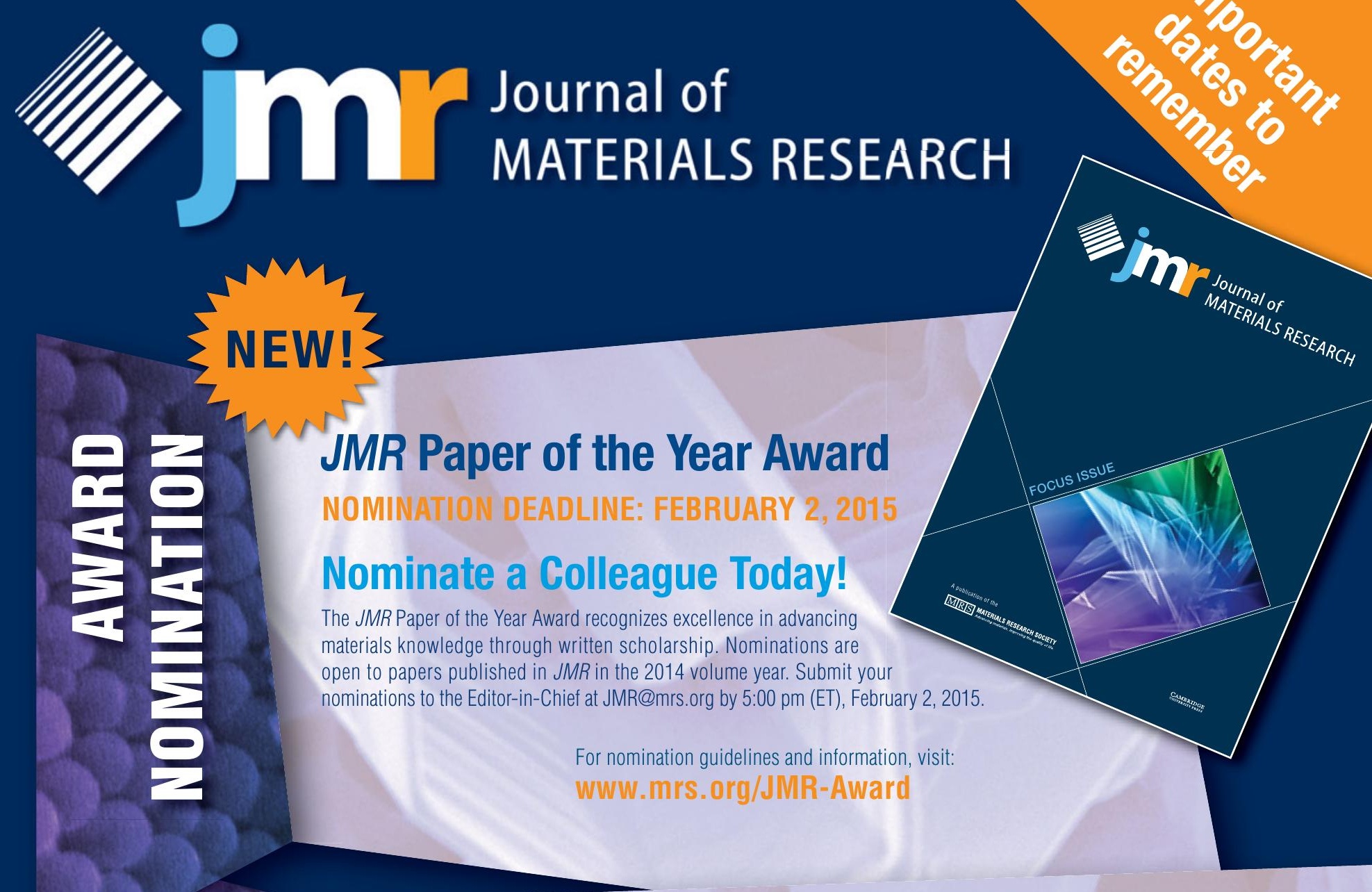

\section{Call For Papers-www.mrs.org/jmr}

\section{Advances in Thermoelectric Materials II}

Submission Deadline

JANUARY 15, 2015

In August of 2011, JMR published "Advances in

Thermoelectric Materials." Since then, there have been significant advances in research such as a surge in thermoelectric nanocomposites, mesoscale systems, and new naturally occurring materials with favorable thermoelectric performance. This JMR Focus Issue will highlight new theoretical ideas, new materials and new device concepts, various processing and synthesis methods, along with technologies and applications.

\section{GUEST EDITORS}

Terry M. Tritt, Clemson University

Harald Böttner, Fraunhofer Institute IPM

Ryoji Funahashi, National Institute of AIST

\section{October 2015 Issue}

Nitrides and Oxynitride Materials

Submission Deadline FEBRUARY 15, 2015

Nitride-based compounds have established themselves as inevitable materials with wide ranging applications in solid state lighting, displays, power electronics, and photovoltaics. Unique features such as polarity can lead to new device modes or improved performance. The convergence of nitride materials and nanomaterials could lead to additional functionalities when combined with organics and polymers. Numerous challenges remain to be resolved in order to fully explore and implement their potential.

\section{GUEST EDITORS}

Zoltan Lences, Slovak Academy of Sciences Tania Paskova, North Carolina State University Joan Redwing, The Pennsylvania State University Rong-Jun Xie, National Institute for Materials Science 


\section{Materials that matter}

The Royal Society of Chemistry is the world's

leading chemistry community, advancing excellence in the chemical sciences.

Our diverse portfolio covers the depth and breadth of materials science in high impact publications.
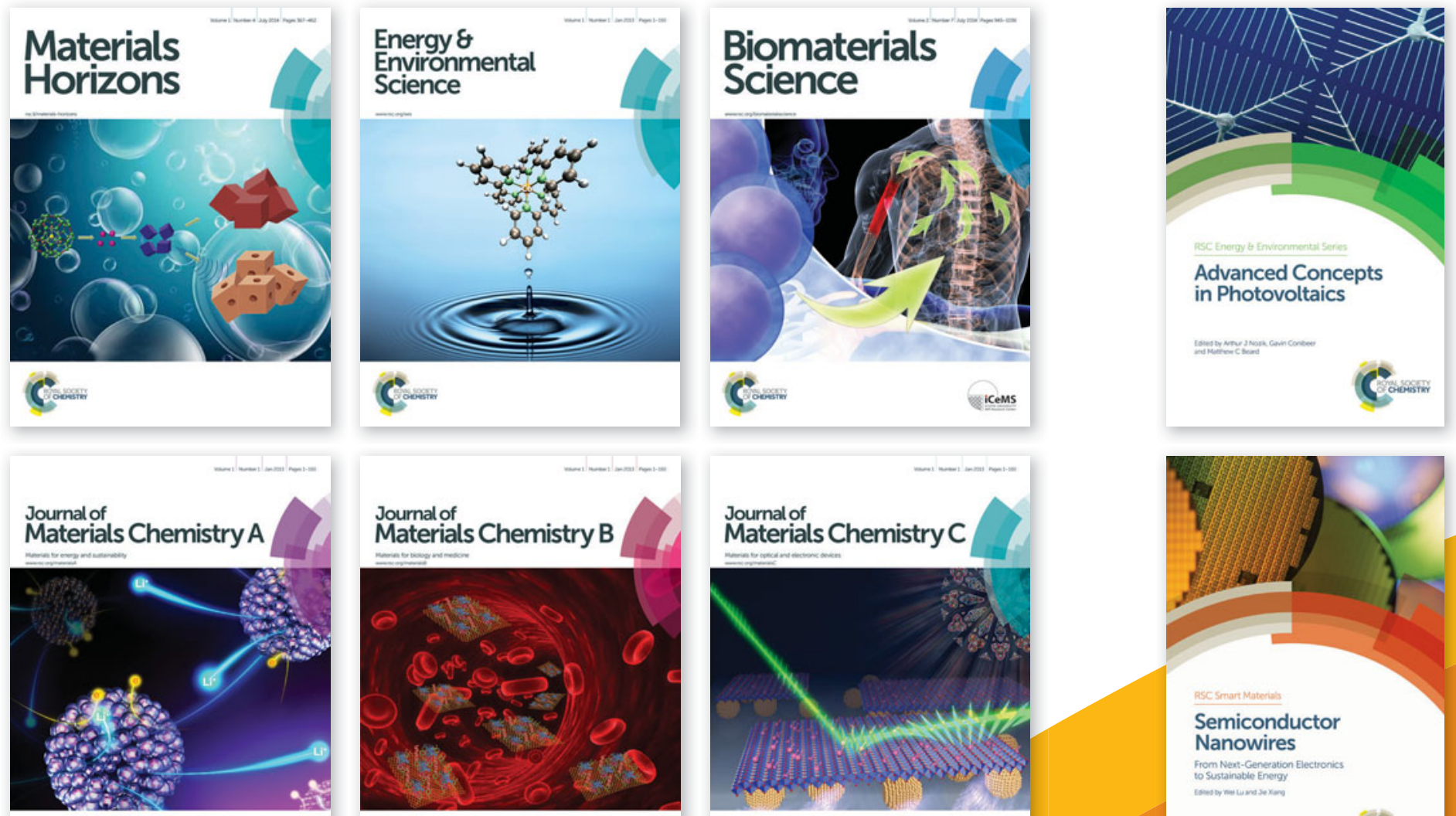

Con
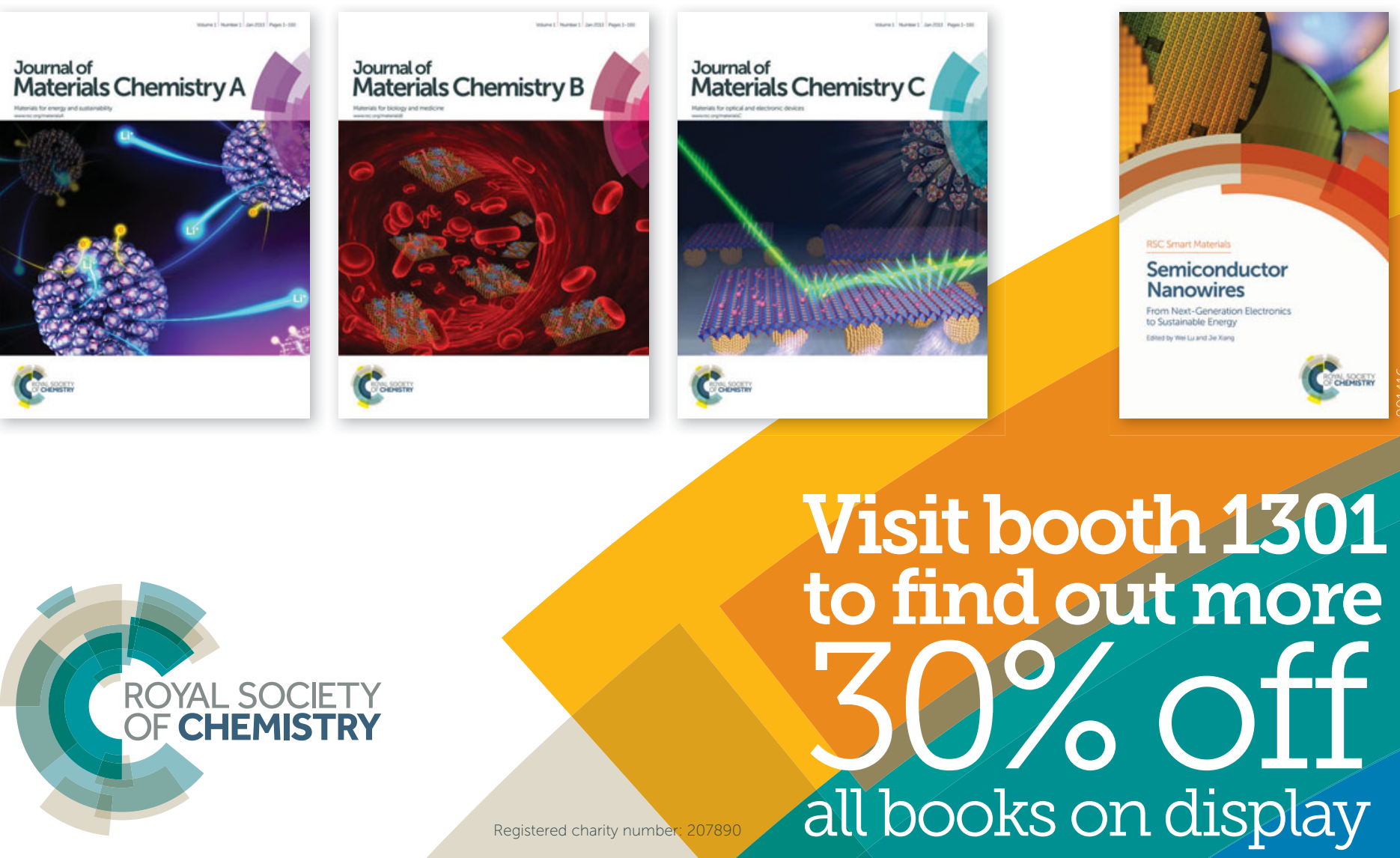